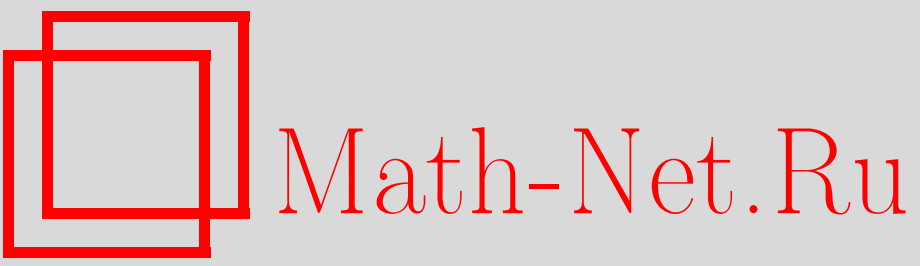

В. И. Солодовников, Три подхода к понятию функций, максимально отличающихся от гомоморфизмов, Матем. вопр. криптогр., 2016, том 7, выпуск 3, 115136

DOI: https://doi.org/10.4213/mvk199

Использование Общероссийского математического портала Math-Net.Ru подразумевает, что вы прочитали и согласны с пользовательским соглашением http://www.mathnet.ru/rus/agreement

Параметры загрузки:

IP : 54.147 .182 .235

26 апреля 2023 г., 18:36:03 
МАТЕМАТИЧЕСКИЕ ВОПРОСЫ КРИПТОГРАФИИ

2016 T. 7 № 3 C. 115-136

УДК 519.719.2

\section{Три подхода к понятию функций, максимально отличающихся от гомоморфизмов}

\section{В. И. Солодовников}

$\mathrm{AO}$ «Институт точной механики и вычислительной техники им. С. А. Лебедева РАН», Москва

Получено 20.IV.2015

Аннотация. Для функций (отображений) из конечной группы в конечную группу рассматриваются три подхода к понятию функций, максимально отличающихся от гомоморфизмов. В основу этих подходов положено понятие «близость функций», альтернативное понятию «расстояние Хэмминга между функциями». Обобщены и развиты понятия абсолютной негомоморфности функции, минимальной близости функции к гомоморфизмам и бент-функции.

Ключевые слова: близость функций, абсолютно негомоморфные функции, максимально негомоморфные функции, минимальные функции, абсолютно минимальные функции, бент-функции, почти бент-функции

Three approaches to the notion of functions maximally differing from homomorphisms

\section{I. Solodovnikov}

Lebedev Institute of Precision Mechanics and Computer Engineering with RAS, JSC, Moscow

Abstract. We consider three approaches to the notion of functions (mappings) from a finite group into a finite group which are maximally differing from homomorphisms. These approaches are based on the notion "functions closeness" which is an alternative to the notion "Hamming distance between functions". The notions of absolute nonhomomorphity of the function, minimal closeness of function to homomorphisms and of bent-function are generalized and studied.

Keywords: functions closeness, absolutely nonhomomorphic functions, maximally nonhomomorphic functions, minimal functions, absolutely minimal functions, bentfunctions, almost bent-functions

Citation: Mathematical Aspects of Cryptography, 2016, v. 7, № 3, pp. 115-136 (Russian) (c) Академия криптографии Российской Федерации, 2016 г. 
В работе используются следующие обозначения и термины.

$\mathbf{X}, \mathbf{Y}$ - нетривиальные конечные группы (с аддитивной записью операций).

$\mathbf{Y}^{\mathbf{X}}-$ множество всех отображений $f: \mathbf{X} \rightarrow \mathbf{Y}$ из множества $\mathbf{X}$ в множество $\mathbf{Y}$ (термины «отображение» и «функция» считаем синонимами).

$\psi \varphi-$ композиция отображений, при которой сначала действует $\varphi$.

$\operatorname{Hom}(G, H)$ - множество всех гомоморфизмов группы $G$ в группу $H$.

$\mathbf{Z}$ - кольцо целых чисел, $\mathbf{Z}_{k}$ - аддитивная группа кольца $\mathbf{Z} /(k)$ вычетов по модулю $k$.

C - поле комплексных чисел, a

$$
\left(\varphi_{1}, \varphi_{2}\right)=\sum_{x \in X} \varphi_{1}(x) \overline{\varphi_{2}(x)}
$$

- стандартное скалярное произведение комплекснозначных функций $\varphi_{1}, \varphi_{2} \in$ $\mathbf{C}^{\mathbf{X}}$.

$P$ - равновероятное вероятностное распределение на $\mathbf{X}$, т. е.

$$
P(M)=|M||\mathbf{X}|^{-1}
$$

для любого $M \subseteq \mathbf{X}$.

Функция $f: \mathbf{X} \rightarrow \mathbf{Y}$ называется сбалансированной, если мощности полных прообразов всех элементов $\mathbf{Y}$ одинаковы. Критерием существования сбалансированных функций является условие

$$
|\mathbf{Y}||| \mathbf{X} \mid
$$

(здесь и далее символ «|» означает «делит»).

Представляется естественным подход к понятию близости функций $f_{1}, f_{2} \in \mathbf{Y}^{\mathbf{X}}$ как величины отличия распределения

$$
P\left(f_{1}-f_{2}=y \mid y \in \mathbf{Y}\right)
$$

от равномерного распределения $\left(|\mathbf{Y}|^{-1}, \ldots,|\mathbf{Y}|^{-1}\right)$. Эти распределения многомерные векторы размерности $|\mathbf{Y}|$. Естественно, возникает задача об определении одномерной числовой характеристики, измеряющей это отличие, т. е. близость. Одну из таких характеристик можно выразить через расстояние (Хэмминга) $\rho$

$$
\rho\left(f_{1}, f_{2}\right)=P\left(f_{1} \neq f_{2}\right)=1-P\left(f_{1}-f_{2}=0\right),
$$


определив в качестве характеристики близости величину

$$
\left.\left|P\left(f_{1}-f_{2}=0\right)-\right| \mathbf{Y}\right|^{-1} \mid .
$$

Для бинарного случая $\mathbf{Y}=\mathbf{Z}_{2}$ этой величины вполне достаточно для измерения близости, поскольку

$$
\left|P\left(f_{1}-f_{2}=0\right)-\frac{1}{2}\right|=\left|P\left(f_{1}-f_{2}=1\right)-\frac{1}{2}\right| .
$$

Однако для $|\mathbf{Y}| \geqslant 3$ по ней уже нельзя оценить величины

$$
\left.\left|P\left(f_{1}-f_{2}=y\right)-\right| \mathbf{Y}\right|^{-1} \mid, \quad 0 \neq y \in \mathbf{Y} .
$$

В [10] предложена другая характеристика, учитывающая и эти величины. Будем рассматривать $\mathbf{Y}$ как пространство элементарных событий с равномерным распределением на нем. Тогда вероятность

$$
P\left(f_{1}-f_{2}=y\right)
$$

как функция от $y \in \mathbf{Y}$ станет случайной величиной; ее математическое ожидание равно $|\mathbf{Y}|^{-1}$. В [10] близость двух произвольных функций $f_{1}, f_{2} \in \mathbf{Y}^{\mathbf{X}}$ было предложено измерять корнем из ее дисперсии

$$
\left(|\mathbf{Y}|^{-1} \sum_{y \in \mathbf{Y}}\left(P\left(f_{1}-f_{2}=y\right)-|\mathbf{Y}|^{-1}\right)^{2}\right)^{1 / 2} .
$$

В [3] предложено нормировать эту величину так, чтобы ее максимальное значение было равно 1, и в качестве меры близости функций рассматривать

$$
\left(|\mathbf{Y}|(|\mathbf{Y}|-1)^{-1} \sum_{y \in \mathbf{Y}}\left(P\left(f_{1}-f_{2}=y\right)-|\mathbf{Y}|^{-1}\right)^{2}\right)^{1 / 2}
$$

Это позволило упростить многие формулы. С целью дальнейших упрощений здесь, как и в $[11,12]$, мы откажемся и от корня.

Определение 1. Мерой близости или, короче, близостью функций $f_{1}, f_{2} \in \mathbf{Y}^{\mathbf{X}}$ назовем число

$$
\delta\left(f_{1}, f_{2}\right)=|\mathbf{Y}|(|\mathbf{Y}|-1)^{-1} \sum_{y \in \mathbf{Y}}\left(P\left(f_{1}-f_{2}=y\right)-|\mathbf{Y}|^{-1}\right)^{2} .
$$


Близость непустых классов функций $K_{1}, K_{2} \subseteq \mathbf{Y}^{\mathbf{X}}$ определим равенством

$$
\delta\left(K_{1}, K_{2}\right)=\max _{\substack{f_{1} \in K_{1} \\ f_{2} \in K_{2}}} \delta\left(f_{1}, f_{2}\right) .
$$

Заметим, что таким образом на множестве $\mathbf{Y}^{\mathbf{X}} \times \mathbf{Y}^{\mathbf{X}}$ мы определили (и назвали «близостью») числовую функцию $\delta$, не зависящую от групповой операции на множестве $\mathbf{X}$. Поэтому определение 1 корректно и в случае, когда $\mathbf{X}$ - произвольное непустое конечное множество (в частности, множество $\mathbf{Y}^{l}$ всех последовательностей одной и той же длины $l$ ).

Легко показать, что близость $\delta$ обладает следующими свойствами:

$$
\begin{gathered}
0 \leqslant \delta\left(f_{1}, f_{2}\right) \leqslant 1, \\
\delta\left(f_{1}, f_{2}\right)=0 \Leftrightarrow f_{1}-f_{2}-\text { сбалансированная, } \\
\delta\left(f_{1}, f_{2}\right)=1 \Leftrightarrow f_{1}-f_{2}=\mathrm{const}, \\
\delta\left(f_{1}, f_{2}\right)=\delta\left(f_{2}, f_{1}\right), \\
\delta\left(y_{1}+g_{2} f_{1} g_{1}+f^{\prime}, y_{2}+g_{2} f_{2} g_{1}+f^{\prime}\right)=\delta\left(f_{1}, f_{2}\right)
\end{gathered}
$$

для любых функций $f_{1}, f_{2} \in \mathbf{Y}^{\mathbf{X}}$, сбалансированной функции $g_{1}: \mathbf{X}^{\prime} \rightarrow \mathbf{X}$, изоморфизма групп $g_{2}: \mathbf{Y} \rightarrow \mathbf{Y}^{\prime}$, функции $f^{\prime}: \mathbf{X}^{\prime} \rightarrow \mathbf{Y}^{\prime}$ и $y_{1}, y_{2} \in \mathbf{Y}^{\prime}$.

Свойства (1)-(3) означают, что минимально близкие функции - это функции, разность которых сбалансированна, а максимально близкие функции это функции, разность которых есть константа.

Представляется естественным величину $\delta(f, 0)$ считать мерой несбалансированности («преобладанием») функции $f$. В частности, критерием сбалансированности функции $f$ является условие

$$
\delta(f, 0)=0 .
$$

Для любого $a \in \mathbf{X}$ подстановку $x \mapsto a+x$ группы $\mathbf{X}$ будем обозначать через $a^{+}$. Все такие подстановки называют сдвигами группы $\mathbf{X}$. Они образуют изоморфную группе $\mathbf{X}$ группу подстановок, называемую группой сдвигов группы $\mathbf{X}$ (в теории представлений групп такое представление группы $\mathbf{X}$ называют регулярным).

Для любого класса функций $K \subseteq \mathbf{Y}^{\mathbf{X}}$ обозначим через

$$
{ }^{+} K=\left\{b^{+} f \mid b \in \mathbf{Y}, f \in K\right\}
$$


множество всех функций из $K$ со сдвигами. В силу (5)

$$
\delta\left(K_{1}, K_{2}\right)=\delta\left({ }^{+} K_{1},{ }^{+} K_{2}\right)
$$

для любых непустых классов функций $K_{1}, K_{2} \subseteq \mathbf{Y}^{\mathbf{X}}$.

Имеются основания считать, что гомоморфизмы абелевых групп со сдвигами (в частности, аффинные отображения векторных пространств) являются конструктивно наиболее простыми функциями. В этой работе рассматриваются три подхода к понятию функций, максимально отличающихся от гомоморфизмов со сдвигами.

Первый подход, который можно назвать «аксиоматическим», основан на невыполнении свойства гомоморфности, т. е. определения (аксиомы) «гомоморфизма». Покажем, что гомоморфизмы со сдвигами характеризуются следующим образом.

Лемма 1. Следующие утверждения равносильны:

1) $f \in \in^{+} \operatorname{Hom}(\mathbf{X}, \mathbf{Y})$;

2) $f a^{+}-f=$ const для всех $a \in \mathbf{X} \backslash\{0\}$.

Доказательство. Имеем:

$f a^{+}-f=$ const для всех $a \in \mathbf{X} \backslash\{0\} \Leftrightarrow$

$\Leftrightarrow f(a+x)-f(x)=f(a)-f(0)$ для всех $a, x \in \mathbf{X} \Leftrightarrow$

$\Leftrightarrow-f(0)+f(a+x)=(-f(0)+f(a))+(-f(0)+f(x))$ для всех $a, x \in \mathbf{X} \Leftrightarrow$

$\Leftrightarrow-f(0)+f \in \operatorname{Hom}(\mathbf{X}, \mathbf{Y}) \Leftrightarrow f \in^{+} \operatorname{Hom}(\mathbf{X}, \mathbf{Y})$.

Лемма доказана.

Сбалансированные функции - это функции, максимально отличающиеся от констант (см. также свойства (1)-(3)). Поэтому в силу леммы 1 естественным является следующее (данное в [10] для случая абелевых групп) определение, совпадающее с определением «совершенной нелинейности» функций для произвольных групп в [8] и функций над кольцом вычетов в [7]. (Далее вместо получившего распространение термина «нелинейность» используется термин «негомоморфность» по следующим причинам: в алгебре линейными отображениями называют отображения векторных пространств, которые, по определению, кроме гомоморфности по сложению, обладают еще и свойством вынесения константы.) 
Определение 2. Функцию $f \in \mathbf{Y}^{\mathbf{X}}$ назовем абсолютно негомоморфной (функцией из группы $\mathbf{X}$ в группу $\mathbf{Y}$ ), если для любого $a \in \mathbf{X} \backslash\{0\}$ функция $f a^{+}-f$ является сбалансированной. Множество всех абсолютно негомоморфных функций из $\mathbf{Y}^{\mathbf{X}}$ обозначим через $A N\left(\mathbf{Y}^{\mathbf{X}}\right)$.

В частности, если $A N\left(\mathbf{Y}^{\mathbf{X}}\right) \neq \varnothing$, то $|\mathbf{Y}||| \mathbf{X} \mid$. Для примарного случая критерий существования абсолютно негомоморфных функций, т. е. условия

$$
A N\left(\mathbf{Y}^{\mathbf{X}}\right) \neq \varnothing,
$$

будет указан в утверждении 7 (с учетом теоремы 2).

Определение 2 можно обобщить следующим образом.

Определение 3. Показателем гомоморфности функции $f \in \mathbf{Y}^{\mathbf{X}}$ назовем число

$$
\delta_{1}(f)=(|\mathbf{X}|-1)^{-1} \sum_{0 \neq a \in \mathbf{X}} \delta\left(f a^{+}, f\right) .
$$

Для любого непустого класса функций $K \subseteq \mathbf{Y}^{\mathbf{X}}$ минимальный показатель гомоморфности функций из $K$ назовем показателем гомоморфности класса $K$ (функций из группы $\mathbf{X}$ в группу $\mathbf{Y}$ ) и обозначим через

$$
\delta_{1}(K)=\min _{f \in K} \delta_{1}(f),
$$

а всякую функцию $f \in K$, для которой $\delta_{1}(f)=\delta_{1}(K)$, назовем максимально негомоморфной в классе $K$ (функций из группы $\mathbf{X}$ в группу $\mathbf{Y})$. Множество всех максимально негомоморфных функций в $K$ обозначим через $N(K)$.

Заметим, что $N(K)=\varnothing$. В частности, в интересном для приложений случае $\mathbf{X}=\mathbf{Y}, \quad K=S_{X}-$ множество всех подстановок множества $\mathbf{X}$ получаем определение «максимально негомоморфной подстановки группы $\mathbf{X}$ ».

Заметим также, что определение максимально негомоморфной функции для $K=\mathbf{Y}^{\mathbf{X}}$ равносильно данному в [8] определению «почти совершенно нелинейной» функции.

Из формул (1)-(3), определения 2 и леммы 1 следует, что для любой $f \in \mathbf{Y}^{\mathbf{X}}$

$$
\begin{gathered}
0 \leqslant \delta_{1}(f) \leqslant 1, \\
\delta_{1}(f)=0 \Leftrightarrow f \in A N\left(\mathbf{Y}^{\mathbf{X}}\right), \\
\delta_{1}(f)=1 \Leftrightarrow f \in^{+} \operatorname{Hom}(\mathbf{X}, \mathbf{Y}),
\end{gathered}
$$

и, следовательно, $\delta_{1}\left(\mathbf{Y}^{\mathbf{X}}\right)=0 \Leftrightarrow A N\left(\mathbf{Y}^{\mathbf{X}}\right) \neq \varnothing \Leftrightarrow A N\left(\mathbf{Y}^{\mathbf{X}}\right)=N\left(\mathbf{Y}^{\mathbf{X}}\right)$. 
Например, для булевой функции $f \in V_{1}^{V_{n}}$ (здесь и далее $V_{k}=\left(\mathbf{Z}_{2}\right)^{k}$ )

$$
\delta_{1}(f)=4\left(2^{n}-1\right) \sum_{0 \neq a \in V_{n}}\left(P\left(f a^{+}=f\right)-\frac{1}{2}\right)^{2} .
$$

Заметим, что альтернативным определению 3 может служить определение, полученное из определения 3 заменой $\delta_{1}(f)$ величиной

$$
\delta_{1}^{\prime}(f)=\max _{0 \neq a \in \mathbf{X}} \delta\left(f a^{+}, f\right) .
$$

Ясно, что $\delta_{1}(f) \leqslant \delta_{1}^{\prime}(f)$.

Как отмечено в [10], к понятию абсолютной (максимальной) негомоморфности можно подойти и с криптографической стороны. А именно, пусть $G$ - некоторая группа подстановок множества $\mathbf{X}, f G=\{f g \mid g \in G\}$ - класс функций, порожденнылй функиией $f \in \mathbf{Y}^{\mathbf{X}}$ и группой $G$. Пусть в некоторой криптосхеме имеется узел, реализующий функции из $f G$, где элементы группы $G$ являются ключами. Тогда величины $\delta\left(f g_{1}, f g_{2}\right), g_{1}, g_{2} \in G$, характеризуют степень изменения этого узла ключами из $G$ : чем ближе эти величины к 0 , тем сильнее подстановки из $G$ изменяют функцию $f$. Если величина $\delta\left(f g_{1}, f g_{2}\right)$ близка к 1 , то ключи $g_{1}$ и $g_{2}$ называют близкими. Так как, в силу (5), $\delta\left(f g_{1}, f g_{2}\right)=\delta\left(f g_{1} f g_{2}^{-1}, f\right)$, то достаточно рассматривать только величины $\delta(f g, f), g \in G$. В частности, когда $G$ - группа сдвигов группы $\mathbf{X}$, получаем, что абсолютно негомоморфные функции - это функции, которые максимально изменяются сдвигами, т. е. соответствующие узлы не имеют близких ключей-сдвигов. В связи с этим рассмотрим величину $P\left(f g=f^{\prime}\right)-|Y|^{-1}$ как случайную величину при равновероятном выборе $g$ из $G$.

В следующих трех утверждениях, обобщающих соответствующие утверждения из [10], используются обозначения:

$X, Y$ - произвольные конечные непустые множества,

$G$ - группа подстановок множества $X$,

$k$ - натуральное число,

$\operatorname{Orb}\left(G, X^{k}\right)-$ множество всех орбит группы $G$, действующей слева на множестве $X^{k}$ по формуле

$$
g\left(x_{1}, x_{2}, \ldots, x_{k}\right)=\left(g\left(x_{1}\right), g\left(x_{2}\right), \ldots, g\left(x_{k}\right)\right),
$$

$f_{1} \times f_{2} \times \cdots \times f_{k}$ - декартово произведение функций $f_{1}, f_{2}, \ldots, f_{k} \in Y^{X}$, T. е. $f_{1} \times f_{2} \times \cdots \times f_{k}\left(x_{1}, x_{2}, \ldots, x_{k}\right)=\left(f_{1}\left(x_{1}\right), f_{2}\left(x_{1}\right), \ldots, f_{k}\left(x_{k}\right)\right)$, $P^{(k)}$ - равновероятное распределение на множестве $X^{k}$. 
Теорема 1. Для любых $f_{1}, \ldots, f_{k}, f_{1}^{\prime}, \ldots, f_{k}^{\prime} \in Y^{X}$

$$
\begin{aligned}
& \frac{1}{|G|} \sum_{g \in G} \prod_{i=1}^{k} P\left(f_{i} g=f_{i}^{\prime}\right)= \\
& =\frac{1}{|X|^{k}} \sum_{T \in \operatorname{Orb}\left(G, X^{k}\right)}|T| \sum_{y \in Y^{k}} P^{(k)}\left(f_{1} \times \cdots \times f_{k}=y \mid T\right) P^{(k)}\left(f_{1}^{\prime} \times \cdots \times f_{k}^{\prime}=y \mid T\right) .
\end{aligned}
$$

Доказательство. Пусть $\Delta-$ символ Кронекера. Тогда

$$
\begin{aligned}
& \frac{1}{|G|} \sum_{g \in G} \prod_{i=1} P\left(f_{i} g=f_{i}^{\prime}\right)= \\
& =\frac{1}{|G||X|^{k}} \sum_{g \in G} \prod_{i=1}^{k} \sum_{y_{i} \in Y} \sum_{x_{i} \in X} \Delta\left(f_{i} g\left(x_{i}\right), y_{i}\right) \Delta\left(f_{i}^{\prime}\left(x_{i}\right), y_{i}\right)= \\
& =\frac{1}{|G||X|^{k}} \sum_{g \in G} \sum_{\substack{\left(y_{1}, \ldots, y_{k}\right) \in Y^{k} \\
\left(x_{1}, \ldots, x_{k}\right) \in X^{k}}} \prod_{i=1}^{k} \Delta\left(f_{i} g\left(x_{i}\right), y_{i}\right) \Delta\left(f_{i}^{\prime}\left(x_{i}\right), y_{i}\right)= \\
& =\frac{1}{|G||X|^{k}} \sum_{\substack{\left(y_{1}, \ldots, y_{k}\right) \in Y^{k} \\
\left(x_{1}, x_{k}\right) \in X^{k}}} \sum_{g \in G} \prod_{i=1}^{k} \Delta\left(f_{i} g\left(x_{i}\right), y_{i}\right) \Delta\left(f_{i}^{\prime}\left(x_{i}\right), y_{i}\right)= \\
& =\frac{1}{|G||X|^{k}} \sum_{\left(y_{1}, \ldots, y_{k}\right) \in Y^{k}}\left(\prod_{i=1}^{k} \Delta\left(f_{i}^{\prime}\left(x_{i}\right), y_{i}\right)\right) \sum_{g \in G} \prod_{i=1}^{k} \Delta\left(f_{i} g\left(x_{i}\right), y_{i}\right)= \\
& \left(x_{1}, \ldots, x_{k}\right) \in X^{k} \\
& =\frac{1}{|G||X|^{k}} \sum_{\left(y_{1}, \ldots, y_{k}\right) \in Y^{k}} \sum_{T \in \operatorname{Orb}\left(G, X^{k}\right)} \sum_{\left(x_{1}, \ldots, x_{k}\right) \in T}\left(\prod_{i=1}^{k} \Delta\left(f_{i}^{\prime}\left(x_{i}\right), y_{i}\right)\right) \times \\
& \times \sum_{g \in G} \prod_{i=1}^{k} \Delta\left(f_{i} g\left(x_{i}\right), y_{i}\right)= \\
& =\frac{1}{|G||X|^{k}} \sum_{\left(y_{1}, \ldots, y_{k}\right) \in Y^{k}} \sum_{T \in \operatorname{Orb}\left(G, X_{k}\right)} \sum_{\left(x_{1}, \ldots, x_{k}\right) \in T}\left(\prod_{i=1}^{k} \Delta\left(f_{i}^{\prime}\left(x_{i}\right), y_{i}\right)\right) \times \\
& \times|G| P^{(k)}\left(f_{1} \times \cdots \times f_{k}=\left(y_{1}, \ldots, y_{k}\right) \mid T\right)= \\
& =\frac{1}{|X|^{k}} \sum_{\left(y_{1}, \ldots, y_{k}\right) \in Y^{k}} \sum_{T \in \operatorname{Orb}\left(G, X^{k}\right)} P^{(k)}\left(f_{1} \times \cdots \times f_{k}=\left(y_{1}, \ldots, y_{k}\right) \mid T\right) \times \\
& \times|T| P^{(k)}\left(f_{1}^{\prime} \times \ldots \times f_{k}^{\prime}=\left(y_{1}, \ldots, y_{k}\right) \mid T\right) .
\end{aligned}
$$


Для $k=1$ получаем два следствия.

Следствие 1. Для любых $f, f^{\prime} \in Y^{X}$

$$
\begin{aligned}
& \frac{1}{|G|} \sum_{g \in G}\left(P\left(f g=f^{\prime}\right)-|Y|^{-1}\right)= \\
& =\frac{1}{|X|} \sum_{T \in \operatorname{Orb}(G)}|T| \sum_{y \in Y}\left(P(f=y \mid T)-|Y|^{-1}\right)\left(P\left(f^{\prime}=y \mid T\right)-|Y|^{-1}\right) .
\end{aligned}
$$

Следствие 2. Если группа $G$ транзитивна, то для любой $f \in Y^{X}$

$$
|G|^{-1} \sum_{g \in G}\left(P(f g=f)-|Y|^{-1}\right)=\sum_{y \in Y}\left(P(f=y)-|Y|^{-1}\right)^{2} .
$$

Отсюда следует, что если $f \in A N\left(\mathbf{Y}^{\mathbf{X}}\right)$, то

$$
\delta(f, 0)=|\mathbf{X}|^{-1} .
$$

Следовательно, абсолютно негомоморфные функции из $\mathbf{Y}^{\mathbf{X}}$ не являются сбалансированными. В частности, абсолютно негомоморфные функиии не могут быть биективными.

Следствие 3. Если группа $\mathbf{Y}$ абелева, то для любых функций $f \in \mathbf{Y}^{\mathbf{X}}$, $h \in \operatorname{Hom}(\mathbf{X}, \mathbf{Y})$

$$
\delta(f, h)=|\mathbf{Y}|(|\mathbf{Y}|-1)^{-1}|\mathbf{X}|^{-1} \sum_{a \in \mathbf{X}}\left(P\left(f a^{+}-f=h(a)\right)-|\mathbf{Y}|^{-1}\right) .
$$

Доказательство. Это равенство вытекает из следствия 2, если в нем в качестве $G$ взять группу сдвигов группы $\mathbf{X}$, а $f$ заменить на $f-h$. Следствие доказано.

Приведем свойства абсолютно негомоморфных функций, вытекающие из следствия 3 и определения 2.

Следствие 4. Если группа $\mathbf{Y}$ абелева, $f \in A N\left(\mathbf{Y}^{\mathbf{X}}\right), h \in \operatorname{Hom}(\mathbf{X}, \mathbf{Y})$, mo

$$
\delta(f, h)=|\mathbf{X}|^{-1}
$$


Следствие 5. Если $f \in A N\left(\mathbf{Y}^{\mathbf{X}}\right), g_{1}: \mathbf{X}^{\prime} \rightarrow \mathbf{X}-$ изоморфизм, $g_{2}: \mathbf{Y} \rightarrow \mathbf{Y}^{\prime}$ - эпиморфизм (т.е. сюръективный гомоморфизм групп), $c \in \mathbf{X}^{\prime}, \quad b \in \mathbf{Y}^{\prime}$, $h^{\prime} \in \operatorname{Hom}\left(\mathbf{X}^{\prime}, \mathbf{Y}^{\prime}\right), \mathbf{Y}^{\prime}$ - абелева группа, то

$$
b+h^{\prime}+g_{2} f g_{1} c^{+} \in A N\left(\mathbf{Y}^{\prime \mathbf{X}^{\prime}}\right) .
$$

В соответствии с теоретико-автоматной терминологией и с учетом групповой структуры алфавитов дадим следующее определение.

Определение 4. Пару $(\alpha, \beta)$ гомоморфизмов $\alpha \in \operatorname{Hom}\left(\mathbf{X}, \mathbf{X}^{\prime}\right), \beta \in \operatorname{Hom}\left(\mathbf{Y}, \mathbf{Y}^{\prime}\right)$ назовем гомоморфизмом функции $f \in \mathbf{Y}^{\mathbf{X}}$ в функцию $f^{\prime} \in \mathbf{Y}^{\prime \mathbf{X}^{\prime}}$, если

$$
\beta f=f^{\prime} \alpha \text {. }
$$

Если $\alpha$ и $\beta$-сюръекции, то гомоморфизм $(\alpha, \beta)$ назовем эпиморфизмом, а функцию $f^{\prime}$-гомоморфным образом функции $f$.

Например, для любых $f \in \mathbf{Y}^{\mathbf{X}}$ и эпиморфизма $\beta \in \operatorname{Hom}\left(\mathbf{Y}, \mathbf{Y}^{\prime}\right)$ функция $\beta f$ - гомоморфный образ функции $f$.

Легко показать, что если $(\alpha, \beta)$ - эпиморфизм функиии $f \in \mathbf{Y}^{\mathbf{X}}$ в функцию $f^{\prime} \in \mathbf{Y}^{\prime \mathbf{X}^{\prime}}$, то для любых $a \in \mathbf{X}$ и $y^{\prime} \in \mathbf{Y}^{\prime}$

$$
P^{\prime}\left(f^{\prime}(\alpha(a))^{+}-f^{\prime}=y^{\prime}\right)-\left|\mathbf{Y}^{\prime}\right|^{-1}=\sum_{y \in \beta^{-1}\left(y^{\prime}\right)}\left(P\left(f a^{+}-f=y\right)-|\mathbf{Y}|^{-1}\right),
$$

где $P^{\prime}\left(M^{\prime}\right)=\left|M^{\prime}\right|\left|\mathbf{X}^{\prime}\right|^{-1}$ для любого $M^{\prime} \subseteq \mathbf{X}^{\prime}$.

Утверждение 1. Если $(\alpha, \beta)$ - эпиморфизм функции $f \in A N\left(\mathbf{Y}^{\mathbf{X}}\right)$ в функичию $f^{\prime} \in \mathbf{Y}^{\prime \mathbf{X}^{\prime}}$ и $\left|\mathbf{Y}^{\prime}\right|>1$, то $\alpha$-изоморфизм и $f^{\prime} \in A N\left(\mathbf{Y}^{\prime \mathbf{X}^{\prime}}\right)$.

Доказательство. Из нетривиальности $\mathbf{Y}^{\prime}$, формулы (6) и абсолютной негомоморфности $f$ следует, что $\alpha$ - изоморфизм. Абсолютная негомоморфность функции $f^{\prime}$ следует из сюръективности $\alpha$, формулы (6) и абсолютной негомоморфности $f$. Утверждение доказано.

Утверждение 2. Пусть $\mathbf{X}_{1}, \mathbf{X}_{2}$ - нетривиальные конечные группы, $f_{i}: \mathbf{X}_{i} \rightarrow \mathbf{Y}$ $(i=1,2), \quad \mathbf{X}=\mathbf{X}_{1} \times \mathbf{X}_{2}, f=f_{1}+f_{2} \in \mathbf{Y}^{\mathbf{X}}$. Тогда

1) если одна из функиий $f_{1}, f_{2}$ сбалансированная, то $f$ сбалансированна;

2) если группа $\mathbf{Y}$ абелева, то

$$
f \in A N\left(\mathbf{Y}^{\mathbf{X}}\right) \Leftrightarrow f_{i} \in A N\left(\mathbf{Y}^{X_{i}}\right), \quad i=1,2 .
$$

Доказательство. Очевидно. 
Второй подход к понятию функций, максимально отличающихся от гомоморфизмов (этот подход можно назвать «метрическим»), состоит в следующем.

Определение 5 ([10]). Близостью функици $f \in \mathbf{Y}^{\mathbf{X}}$ к гомоморфизмам назовем число

$$
\delta_{2}(f)=\delta(f, \operatorname{Hom}(\mathbf{X}, \mathbf{Y})) .
$$

Для любого непустого класса функций $K \subseteq \mathbf{Y}^{\mathbf{X}}$ минимальную близость $\kappa$ гомоморфизмам функций из $K$ обозначим через

$$
\delta_{2}(K)=\min _{f \in K} \delta_{2}(f)
$$

а всякую функцию $f \in K$, для которой

$$
\delta_{2}(f)=\delta_{2}(K)
$$

назовем минимально близкой к гомоморфизмам или, короче, минимальной функцией в классе $K$ (функций из группы $\mathbf{X}$ в группу $\mathbf{Y})$. Множество всех минимальных функций в $K$ обозначим через $M(K)$.

Заметим, что $M(K) \neq \varnothing$. В частности, в случае

$$
\mathbf{X}=\mathbf{Y}, K=S_{X}-\text { множество всех подстановок множества } \mathbf{X} \text {, }
$$

интересном для приложений, получаем определение «минимальной подстановки группы $\mathbf{X} »$.

Из формул (1) и (3) следует, что для любой $f \in \mathbf{Y}^{\mathbf{X}}$

$$
\begin{gathered}
\delta_{2}(f) \leqslant 1, \\
\delta_{2}(f)=1 \Leftrightarrow f \in^{+} \operatorname{Hom}(\mathbf{X}, \mathbf{Y}),
\end{gathered}
$$

и если $f \in A N\left(\mathbf{Y}^{\mathbf{X}}\right)$ и группа $\mathbf{Y}$ абелева, то по следствию 4

$$
\delta_{2}(f)=|\mathbf{X}|^{-1}
$$

Заметим, что все вышеприведенные результаты развивают и обобщают соответствующие результаты из $[10,11]$ на случай произвольных (не обязательно абелевых, как в $[10,11])$ групп. 
Прежде чем перейти к третьему подходу к понятию функций, максимально отличающихся от гомоморфизмов (этот подход можно назвать «инструментальным»), введем аппарат (т. е. соответствующий «инструментарий»), используемый во многих работах, посвященных дискретным функциям.

Пусть всюду далее $\mathbf{X}, \mathbf{Y}$ - абелевы группы. Любая абелева группа изоморфна прямому произведению циклических групп. Поэтому, учитывая формулу (5), без ограничения общности далее считаем, что

$$
\mathbf{X}=\prod_{i=1}^{n} \mathbf{Z}_{k_{i}}, \mathbf{Y}=\prod_{j=1}^{m} \mathbf{z}_{t_{j}}
$$

где $n, m$ - произвольные натуральные числа, $k_{1}, k_{2}, \ldots, k_{n}, t_{1}, t_{2}, \ldots, t_{m}$ произвольные натуральные числа, бо́льшие 1. Далее используются обозначения и терминология из [10].

Для каждого $\alpha=\left(\alpha_{1}+\left(k_{1}\right), \alpha_{2}+\left(k_{2}\right), \ldots, \alpha_{n}+\left(k_{n}\right)\right) \in \mathbf{X}$ определим комплекснозначную функцию $\chi_{\alpha}: \mathbf{X} \rightarrow \mathbf{C}$ выражениями

$$
\chi_{\alpha}(x)=\prod_{j=1}^{n} \exp \left\{\frac{2 \pi i}{k_{j}} a_{j} x_{j}\right\}=\exp \left\{2 \pi i \sum_{j=1}^{n} \frac{1}{k_{j}} a_{j} x_{j}\right\}
$$

для всех $x=\left(x_{1}+\left(k_{1}\right), x_{2}+\left(k_{2}\right), \ldots, x_{n}+\left(k_{n}\right)\right) \in \mathbf{X}$.

Из теории представлений групп (см., например, [5]) следует, что $\left\{\chi_{\alpha} \mid \alpha \in \mathbf{X}\right\}$ - множество всех неприводимых характеров абелевой группы $\mathbf{X}$ и выполняются известные соотношения ортогональности

$$
|\mathbf{X}|^{-1}\left(\chi_{\alpha}, \chi_{\alpha^{\prime}}\right)=\Delta\left(\alpha, \alpha^{\prime}\right) \quad \text { для любых } \quad \alpha, \alpha^{\prime} \in \mathbf{X}
$$

( $\Delta$ - символ Кронекера). Свойство (8) означает, что множество $\left\{\chi_{\alpha} \mid \alpha \in \mathbf{X}\right\}$ является ортонормированным относительно скалярного произведения $|\mathbf{X}|^{-1}(\cdot, \cdot)$ базисом векторного пространства $\mathbf{C}^{\mathbf{X}}$, и, следовательно, любая функция $\varphi: \mathbf{X} \rightarrow \mathbf{C}$ однозначно представляется в виде

$$
\varphi=\sum_{\alpha \in \mathbf{X}} C_{\alpha}^{\varphi} \chi_{\alpha}
$$

где $C_{\alpha}^{\varphi} \in \mathbf{C}$ и

$$
C_{\alpha}^{\varphi}=|\mathbf{X}|^{-1}\left(\varphi, \chi_{\alpha}\right) \quad \text { для всех } \quad \alpha \in \mathbf{X} .
$$


Равенство (9) называют разложением Фурье функции $\varphi$, а коэффициенты $C_{\alpha}^{\varphi}, \alpha \in \mathbf{X},-$ коэффициентами Фурье функции $\varphi$. Они однозначно определяют саму функцию $\varphi$ и обладают следующими свойствами:

$$
\begin{gathered}
\left|C_{\alpha}^{\varphi}\right| \leqslant \max _{x \in \mathbf{X}}|\varphi(x)|, \\
C_{0}^{\varphi}=\mathbf{M} \varphi \quad(\text { среднее значение } \varphi), \\
\sum_{\alpha \in \mathbf{X}}\left|C_{\alpha}^{\varphi}\right|^{2}=\mathbf{M}|\varphi|^{2} .
\end{gathered}
$$

Равенство (13) называют равенством Парсеваля.

Пусть всюду далее $\left\{\chi_{\alpha} \mid \alpha \in \mathbf{X}\right\}$ и $\left\{\eta_{\beta} \mid \beta \in \mathbf{Y}\right\}$ - множества всех неприводимых характеров абелевых групп $\mathbf{X}$ и $\mathbf{Y}$ соответственно, определяемые формулой (7).

Определение 6 ([10]). Характерами функции $f \in \mathbf{Y}^{\mathbf{X}}$ назовем функции $\eta_{\beta} f: \mathbf{X} \rightarrow \mathbf{C}$ для всех $\beta \in \mathbf{Y}$.

При $\beta=e_{j}=\left(\left(t_{1}\right), \ldots,\left(t_{j-1}\right), 1+\left(t_{j}\right),\left(t_{j+1}\right), \ldots,\left(t_{m}\right)\right), j \in\{1, \ldots, m\}$, назовем их базисными.

Базисные характеры функции однозначно определяют саму функцию.

Определение 7 ([1]). Производящей функиией функции $f \in \mathbf{Y}^{\mathbf{X}}$ называется функция $\varphi_{f}: \mathbf{Y} \rightarrow \mathbf{C}$, определяемая равенством

$$
\varphi_{f}(\beta)=\mathbf{M} \eta_{\beta} f=|\mathbf{X}|^{-1} \sum_{x \in \mathbf{X}} \eta_{\beta}(f(x)) .
$$

Очевидно, что

$$
\varphi_{f}=\sum_{y \in \mathbf{Y}} P(f=y) \eta_{y}
$$

и, в силу (10),

$$
P(f=y)=|\mathbf{Y}|^{-1}\left(\varphi_{f}, \eta_{y}\right) \quad \text { для любого } y \in \mathbf{Y} .
$$

Следовательно, по производящей функции $\varphi_{f}$ однозначно определяется распределение случайной величины $f$. В частности,

$$
f \text {-сбалансированная } \Leftrightarrow \eta_{f}(\beta)=0 \text { для любого } \beta \neq 0 .
$$

В силу (9) и (10) разложение Фурье характера функции $f$ имеет вид

$$
\eta_{\beta} f=\sum_{\alpha \in X} C_{\alpha}^{\eta_{\beta} f} \chi_{\alpha}, \quad C_{\alpha}^{\eta_{\beta} f}=|\mathbf{X}|^{-1}\left(\eta_{\beta} f, \chi_{\alpha}\right) .
$$


Отсюда следует, что коэффищиенты Фурье базисных характеров функции $f$

$$
C_{\alpha}^{\eta_{\varepsilon_{j}} f}, \quad j=1,2, \ldots, m, \quad a \in \mathbf{X}
$$

однозначно определяют саму функцию $f$ (всего этих чисел $m|\mathbf{X}|$ ). Из (11)(13) получаем следующие свойства:

$$
\begin{gathered}
\left|C_{\alpha}^{\eta_{\beta} f}\right| \leqslant 1, \\
C_{0}^{\eta_{\beta}}=\varphi_{f}(\beta), \\
\sum_{\alpha \in X}\left|C_{\alpha}^{\eta_{\beta} f}\right|^{2}=1 \text { (равенство Парсеваля). }
\end{gathered}
$$

Кроме того, очевидны равенства

$$
\begin{gathered}
\varphi_{f_{1}-f_{2}}(\beta)=\sum_{\alpha \in \mathbf{X}} C_{\alpha}^{\eta_{\beta} f_{1}} \cdot \overline{C_{\alpha}^{\eta_{\beta} f_{2}}}, \\
\delta\left(f_{1}, f_{2}\right)=(|\mathbf{Y}|-1)^{-1} \sum_{0 \neq \beta \in \mathbf{Y}}\left|\varphi_{f_{1}-f_{2}}(\beta)\right|^{2}, \\
C_{\alpha}^{\eta_{\beta}\left(b+f a^{+}\right)}=\eta_{\beta}(b) \chi_{\alpha}(a) C_{\alpha}^{\eta_{\beta} f} \quad \text { для любых } \quad a \in \mathbf{X}, b \in \mathbf{Y}, \\
\varphi_{f a_{1}^{+}-f a_{2}^{+}}(\beta)=\sum_{\alpha \in \mathbf{X}}\left|C_{\alpha}^{\eta_{\beta} f}\right|^{2} \chi_{\alpha}\left(a_{1}-a_{2}\right) \quad \text { для любых } \quad a_{1}, a_{2} \in \mathbf{X} .
\end{gathered}
$$

Утверждение 3 ([10]). Для любых $f_{1}, f_{2} \in \mathbf{Y}^{\mathbf{X}}$ следуюшие утверждения равносильны:

1) $P\left(f_{1} a^{+}-f_{1}=y\right)=P\left(f_{2} a^{+}-f_{2}=y\right)$ для любых $a \in \mathbf{X}, y \in \mathbf{Y}$;

2) $\left|C_{\alpha}^{\eta_{\beta} f_{1}}\right|=\left|C_{\alpha}^{\eta_{\beta} f_{2}}\right|$ для любых $\alpha \in \mathbf{X}, \beta \in \mathbf{Y}$.

Утверждение 3 - прямое следствие (14) и (23).

Следующая лемма - это критерий гомоморфности со сдвигом в терминах коэффициентов Фурье.

Лемма 2. Для любой $f \in \mathbf{Y}^{\mathbf{X}}$ следующие утверждения равносильны:

1) $f \in^{+} \operatorname{Hom}(\mathbf{X}, \mathbf{Y})$;

2) для любого $\beta \in \mathbf{Y} \backslash\{0\}$ существует такой $\alpha \in \mathbf{X}$, что $\left|C_{\alpha}^{\eta_{\beta} f}\right|=1$. 
Доказательство. 1) $\Rightarrow$ 2). Пусть $f=b+h$, где $b \in \mathbf{Y}, h \in \operatorname{Hom}(\mathbf{X}, \mathbf{Y})$. Тогда $\eta_{\beta} f=\eta_{\beta}(b) \eta_{\beta} h$. Так как $\eta_{\beta} h \in \operatorname{Hom}(\mathbf{X}, \mathbf{C}), \eta_{\beta} h=\chi_{\alpha}$ для некоторого $\alpha \in \mathbf{X}$, и 2) доказано.

$2) \Rightarrow 1$ ). Из равенств (21), (23) и (19) следует, что для любого $a \in \mathbf{X}$

$$
\begin{aligned}
\delta\left(f a^{+}, f\right) & =(|\mathbf{Y}|-1)^{-1} \sum_{0 \neq \beta \in \mathbf{Y}}\left|\varphi_{f a^{+}-f}(\beta)\right|^{2}= \\
& =\left.\left.(|\mathbf{Y}|-1)^{-1} \sum_{0 \neq \beta \in \mathbf{Y}}\left|\sum_{\alpha \in \mathbf{X}}\right| C_{\alpha}^{\eta_{\beta} f}\right|^{2} \chi_{\alpha}(a)\right|^{2}=1,
\end{aligned}
$$

что по лемме 1 равносильно утверждению 1). Лемма доказана.

Из (17) и равенства Парсеваля (19) получаем следствие 6.

Следствие 6. Для любых $f \in \mathbf{Y}^{\mathbf{X}}, \beta \in \mathbf{Y} \backslash\{0\}$

$$
|\mathbf{X}|^{-1} \leqslant \max _{a \in X}\left|C_{\alpha}^{\eta_{\beta} f}\right|^{2} \leqslant 1
$$

причем следующие утверждения равносильны:

1) $\max _{a \in X}\left|C_{\alpha}^{\eta_{\beta} f}\right|^{2}=|\mathbf{X}|^{-1}$;

2) $\left|C_{\alpha}^{\eta_{\beta} f}\right|^{2}=|\mathbf{X}|^{-1}$ для всех $a \in \mathbf{X}$;

3) $\left|C_{\alpha}^{\eta_{\beta} f}\right|=\left|C_{\alpha^{\prime}}^{\eta_{\beta} f}\right|$ для любых $\alpha, \alpha^{\prime} \in \mathbf{X}$.

Следствие 6 вместе с леммой 2 обосновывают третий подход к понятию функций, максимально отличающихся от гомоморфизмов («инструментальный» подход). Он описан в следующем определении.

Определение $\mathbf{8}$ ([10]). Функцию $f \in \mathbf{Y}^{\mathbf{X}}$ назовем бент-функиией (из группы $\mathbf{X}$ в группу $\mathbf{Y}$ ), если для любых $\alpha, \alpha^{\prime} \in \mathbf{X}, \beta \in \mathbf{Y} \backslash\{0\}$

$$
\left|C_{\alpha}^{\eta_{\beta} f}\right|=\left|C_{\alpha^{\prime}}^{\eta_{\beta} f}\right| \text {. }
$$

Множество всех бент-функций из группы $\mathbf{X}$ в группу $\mathbf{Y}$ обозначим через $A B\left(\mathbf{Y}^{\mathbf{X}}\right)$.

Это определение обобщает определение работы [9] (для булевых функций, т. е. для $m=1, k_{1}=k_{2}=\cdots=k_{n}=t_{1}=2$ ), определение работы [1] (для $k_{1}=k_{2}=\cdots=k_{n}=t_{1}=\cdots=t_{m}=p$, где $p$ - простое число, $m$ делит $n$ ) и может быть сведено к определению работы [6], где бент-функции определяются как комплекснозначные функции на конечной абелевой группе с единичными модулями всех значений и условием равенства модулей всех коэффициентов Фурье. 
Для случая $m=1, k_{1}=k_{2}=\cdots=k_{n}=t_{1} \geqslant 3$ определение 8 сужает определение бент-функции в [4] (где участвует только базисный характер группы $\mathbf{Y}$ ), но в [4] доказано, что при простом $t_{1}$ эти определения равносильны. В [13] приводится обстоятельный обзор бент-тематики.

В [9] для булевых функций доказано, что классы абсолютно негомоморфных функций и бент-функций совпадают:

$$
A N\left(V_{1}^{V_{n}}\right)=A B\left(V_{1}^{V_{n}}\right) .
$$

Легко показать, что для любой функции $f \in V_{1}^{V_{n}}$ и любой линейной функции $h\left(x_{1}, x_{2}, \ldots, x_{n}\right)=\alpha_{1} x_{1} \oplus \alpha_{2} x_{2} \oplus \cdots \oplus \alpha_{n} x_{n} \in \operatorname{Hom}\left(V_{n}, V_{1}\right)$,

$$
\begin{gathered}
2\left(P(f=h)-\frac{1}{2}\right)=C_{\alpha}^{\eta_{1} f}, \\
\delta(f, h)=\left(C_{\alpha}^{\eta_{1} f}\right)^{2},
\end{gathered}
$$

где $\alpha=\left(\alpha_{1}, \alpha_{2}, \ldots, \alpha_{n}\right)$. Поэтому для булевых функций от четного числа переменных $n$ (для нечетных $n$ булевых бент-функций не существует)

$$
M\left(V_{1}^{V_{n}}\right)=A B\left(V_{1}^{V_{n}}\right),
$$

и, следовательно,

$$
A N\left(V_{1}^{V_{n}}\right)=M\left(V_{1}^{V_{n}}\right)=A B\left(V_{1}^{V_{n}}\right)
$$

т. е. все три подхода совпадают.

В следующей теореме утверждается, что и в общем случае

$$
A N\left(\mathbf{Y}^{\mathbf{X}}\right)=A B\left(\mathbf{Y}^{\mathbf{X}}\right)
$$

т. е. соответствующие результаты из [9], [1] обобщаются на случай произвольных конечных абелевых групп $\mathbf{X}$ и $\mathbf{Y}$.

Tеорема 2 ([10]). Для любой $f \in \mathbf{Y}^{\mathbf{X}}$ следуюшие свойства равносильны:

1) $f$-абсолютно негомоморфная функиия;

2) $f$ - бент-функция;

3) $\left\{\eta_{\beta} f a^{+} \mid a \in \mathbf{X}\right\}-$ ортонормированный относительно скалярного произведения $|\mathbf{X}|^{-1}(\cdot, \cdot)$ базис пространства $\mathbf{C}^{\mathbf{X}}$ для любого $\beta \in \mathbf{Y} \backslash\{0\}$. 
Равенство Парсеваля (19) позволяет смотреть на набор чисел

$$
\left(\left|C_{\alpha}^{\eta_{\beta} f}\right|^{2} \mid \alpha \in \mathbf{X}\right)
$$

как на некоторое вероятностное распределение на множестве $\mathbf{X}$. По лемме 2 гомоморфность со сдвигом функции $f$ равносильна вырожденности этого распределения (одна вероятность равна 1, а остальные равны 0) для любого $\beta \in \mathbf{Y} \backslash\{0\}$. От вырожденных распределений максимально отличается равновероятное (сбалансированное) распределение

$$
\left(|\mathbf{X}|^{-1} \mid \alpha \in \mathbf{X}\right)
$$

Эти рассуждения приводят к следующему определению, аналогичному определению 3 и обобщающему понятие бент-функции.

Определение 9. Показателем несбалансированности коэффициентов Фурье функции $f \in \mathbf{Y}^{\mathbf{X}}$ назовем число

$$
\delta_{3}(f)=|\mathbf{X}|(|\mathbf{X}|-1)^{-1} \sum_{0 \neq \beta \in \mathbf{Y}} \sum_{\alpha \in \mathbf{X}}\left(\left|C_{\alpha}^{\eta_{\beta} f}\right|^{2}-|\mathbf{X}|^{-1}\right)^{2} .
$$

Для любого непустого класса функций $K \subseteq \mathbf{Y}^{\mathbf{X}}$ минимальный показатель несбалансированности коэффициентов Фурье функций из $K$ обозначим через

$$
\delta_{3}(K)=\min _{f \in K} \delta_{3}(f),
$$

а всякую функцию $f \in K$, для которой

$$
\delta_{3}(f)=\delta_{3}(K),
$$

назовем почти бент- функцией в классе $K$ (функций из группы $\mathbf{X}$ в группу $\mathbf{Y})$. Множество всех почти бент-функций в $K$ обозначим через $B(K)$.

Заметим, что альтернативным этому определению является определение «максимально нелинейной функции» в [8], где для случая $K=\mathbf{Y}^{\mathbf{X}}$ вместо $\delta_{3}(f)$ взята величина

$$
\delta_{3}^{\prime}(f)=\max _{\alpha \in \mathbf{X}, \beta \in \mathbf{Y} \backslash\{0\}}\left|C_{\alpha}^{\eta_{\beta} f}\right|^{2} .
$$

Из формул (21) и (22) следует неравенство

$$
\delta_{2}(f) \leqslant \delta_{3}^{\prime}(f),
$$

которое для булевых функций обращается в равенство (см. формулу (25)). 
Следующая теорема показывает, что определение 9 - это интерпретация определения 3 (для случая абелевых групп) в терминах коэффициентов Фурье. Эта теорема обобщает теорему 2 в части совпадения понятий абсолютно негомоморфной функции и бент-функции.

Теорема 3. Для любых $f \in \mathbf{Y}^{\mathbf{X}}, \varnothing \neq K \subseteq \mathbf{Y}^{\mathbf{X}}$ выполняются следуюшие равенства:

1) $\delta_{1}(f)=\delta_{3}(f)$;

2) $\delta_{1}(K)=\delta_{3}(K)$;

3) $N(K)=B(K)$;

4) $A N\left(\mathbf{Y}^{\mathbf{X}}\right)=A B\left(\mathbf{Y}^{\mathbf{X}}\right)$.

Доказательство. Из формул (21), (23) и (8) получаем

$$
|\mathbf{X}|^{-1} \sum_{a \in \mathbf{X}} \delta\left(f a^{+}, f\right)=(|\mathbf{Y}|-1)^{-1} \sum_{0 \neq \beta \in \mathbf{Y}} \sum_{\alpha \in \mathbf{X}}\left|C_{\alpha}^{\eta_{\beta} f}\right|^{4}
$$

Отсюда и из (19) следует, что

$$
\begin{aligned}
& (|\mathbf{Y}|-1)^{-1} \sum_{0 \neq \beta \in \mathbf{Y}} \sum_{\alpha \in \mathbf{X}}\left(\left|C_{\alpha}^{\eta_{\beta} f}\right|^{2}-|\mathbf{X}|^{-1}\right)^{2}= \\
& =(|\mathbf{Y}|-1)^{-1} \sum_{0 \neq \beta \in \mathbf{Y}} \sum_{\alpha \in \mathbf{X}}\left(\left|C_{\alpha}^{\eta_{\beta} f}\right|^{4}-2\left|C_{\alpha}^{\eta_{\beta} f}\right|^{2}|\mathbf{X}|^{-1}+|\mathbf{X}|^{-2}\right)= \\
& =|\mathbf{X}|^{-1} \sum_{\alpha \in \mathbf{X}} \delta\left(f a^{+}, f\right)+(|\mathbf{Y}|-1)^{-1} \sum_{0 \neq \beta \in \mathbf{Y}}\left(-2|\mathbf{X}|^{-1}+|\mathbf{X}|^{-1}\right)= \\
& =|\mathbf{X}|^{-1} \sum_{0 \neq a \in \mathbf{X}} \delta\left(f a^{+}, f\right),
\end{aligned}
$$

и равенство 1) доказано. Остальные равенства следуют из равенства 1), определений $3,9,2,8$ и следствия 6 . Теорема доказана.

Заметим, что равенство 3) для $K=\mathbf{Y}^{\mathbf{X}}$ равносильно теореме 5 из [8].

Следующее утверждение распространяет известный результат работы [2] для булевых функций на случай абелевых групп (с некоторым ограничением). Оно означает, что набор расстояний Хэмминга от функции $f$ до всех гомоморфизмов со сдвигами однозначно определяет функцию $f$ (для булевых функций это следует из равенства (24)). 
Утверждение 4 ([10]). Пусть $k_{i} \mid t_{j}$ для любых $i=1, \ldots, n, j=1, \ldots, m$. Тогда для любой $f \in \mathbf{Y}^{\mathbf{X}}$ набор чисел

$$
\left(P(f=h) \mid h \in^{+} \operatorname{Hom}(\mathbf{X}, \mathbf{Y})\right)
$$

однозначно определяет функцию $f$.

Следующие два утверждения сводят многомерный случай к одномерному и обобщают теорему 3 из [1].

Утверждение 5 ([10]). Для случая $\mathbf{X}=\left(\mathbf{Z}_{t}\right)^{n}, \mathbf{Y}=\left(\mathbf{Z}_{t}\right)^{m}$ и любой $f \in \mathbf{Y}^{\mathbf{X}}$ следующие свойства равносильны:

1) $f$ - сбалансированная функиия;

2) $h f-$ сбалансированная функиия для любого эпиморфизма $h: \mathbf{Y} \rightarrow \mathbf{Z}_{t}$.

Утверждение 6 ([10]). Для любой $f \in \mathbf{Y}^{\mathbf{X}}$ следующие свойства равносильны:

1) $f$ - бент-функция;

2) $h f$ - бент-функция для любого эпиморфизма $h: \mathbf{Y} \rightarrow \mathbf{Z}_{t}$.

Далее рассмотрим случай, названный в $[11,12]$ примарным. Особенность этого случая характеризуется следующей леммой.

Лемма 3 ([11]). Следующие свойства равносильны:

a) для любых $x \in \mathbf{X} \backslash\{0\}$ существует такой $h \in \operatorname{Hom}(\mathbf{X}, \mathbf{Y})$, что $h(x)=y$;

б) $k_{1}=k_{2}=\cdots=k_{n}=t_{1}=t_{2}=\cdots=t_{m}=p$, где $p$ - простое число.

Условие а) существенно для дальнейших рассуждений (лемма 4). Поэтому далее рассмотрим только случай выполнения условия б) леммы 3 :

$$
\mathbf{X}=\left(\mathbf{Z}_{p}\right)^{n}, \quad \mathbf{Y}=\left(\mathbf{Z}_{p}\right)^{m}, \quad p-\text { простое }, \quad p>1,
$$

который назовем примарнымм (или $p$-примарным), а функции из $\mathbf{Y}^{\mathbf{X}}-n p u$ марными (или $p$-примарными). В этом случае гомоморфизмы из $\operatorname{Hom}(\mathbf{X}, \mathbf{Y})$ являются линейными функциями (гомоморфизмами векторных пространств над полем $\mathbf{Z} /(p)$ ). 2-примарный случай называют двоичным. Двоичный случай для $m=1$ называют булевым. С практической точки зрения оба эти случая наиболее интересны.

Приведем критерий существования примарных бент-функций, полученный применением результатов из [7,1], утверждения 1 и теоремы 2. 
Утверждение 7 ([12]). Для р-примарного случая следующие утверждения равносильны:

1) $A B\left(\mathbf{Y}^{\mathbf{X}}\right) \neq \varnothing$;

2) выполняется одно из следующих условий:

$$
\begin{array}{ll}
p=2, & 2 \mid n, \quad n \geqslant 2 m, \\
p \geqslant 3, & n \geqslant m .
\end{array}
$$

Лемма 4 ([10]). Для любой примарной $f: \mathbf{X} \rightarrow \mathbf{Y}$

$$
|\mathbf{Y}|^{-n} \sum_{h \in \operatorname{Hom}(\mathbf{X}, \mathbf{Y})} \delta(f, h)=|\mathbf{X}|^{-1} .
$$

Из этой леммы получаем оценку минимальной близости примарных функций к линейным функциям.

Утверждение 8 ([10]). Для примарного случая $\delta_{2}\left(\mathbf{Y}^{\mathbf{X}}\right) \geqslant|\mathbf{X}|^{-1}$.

Отсюда естественным образом возникает следующее определение.

Определение 10 ([11]). В случае выполнения условия $\delta_{2}\left(\mathbf{Y}^{\mathbf{X}}\right)=|\mathbf{X}|^{-1}$ примарные минимальные функции в $\mathbf{Y}^{\mathbf{X}}$ назовем абсолютно минимальными.

Обозначим $A M\left(\mathbf{Y}^{\mathbf{X}}\right)$ множество всех абсолютно минимальных функций.

Таким образом,

$$
A M\left(\mathbf{Y}^{\mathbf{X}}\right)=\left\{\begin{array}{lll}
M\left(\mathbf{Y}^{\mathbf{X}}\right), & \text { если } & \delta_{2}\left(\mathbf{Y}^{\mathbf{X}}\right)=|\mathbf{X}|^{-1} \\
\varnothing, & \text { если } & \delta_{2}\left(\mathbf{Y}^{\mathbf{X}}\right)>|\mathbf{X}|^{-1}
\end{array}\right.
$$

Утверждение 9 ([10]). В примарном случае $A N\left(\mathbf{Y}^{\mathbf{X}}\right) \subseteq A M\left(\mathbf{Y}^{\mathbf{X}}\right)$.

Утверждение 9 вытекает из следствия 4, утверждения 8 определений 5 и 10 .

Следствие 7. Для примарного случая следующие утверждения равносильны:

1) $A B\left(\mathbf{Y}^{\mathbf{X}}\right) \neq \varnothing$

2) $0=\delta_{1}\left(\mathbf{Y}^{\mathbf{X}}\right)=\delta_{1}^{\prime}\left(\mathbf{Y}^{\mathbf{X}}\right)=\delta_{2}\left(\mathbf{Y}^{\mathbf{X}}\right)-|\mathbf{X}|^{-1}=\delta_{3}^{\prime}\left(\mathbf{Y}^{\mathbf{X}}\right)-|\mathbf{X}|^{-1}$,

$$
A N\left(\mathbf{Y}^{\mathbf{X}}\right)=N\left(\mathbf{Y}^{\mathbf{X}}\right)=B\left(\mathbf{Y}^{\mathbf{X}}\right)=A B\left(\mathbf{Y}^{\mathbf{X}}\right) \subseteq A M\left(\mathbf{Y}^{\mathbf{X}}\right)=M\left(\mathbf{Y}^{\mathbf{X}}\right) .
$$

Следствие 7 несложно вывести из утверждения 9, теоремы 2, следствия 4 и определений $3,5,9,10$.

В следующей лемме собраны критерии абсолютной минимальности примарных функций. 
Лемма 5 ([11]). Для любой р-примарной $f: \mathbf{X} \rightarrow \mathbf{Y}$ следуюшие утверждения равносильны:

1) $f \in A M\left(\mathbf{Y}^{\mathbf{X}}\right)$;

2) $\delta(f, h)=|\mathbf{X}|^{-1}$ для $h \in \operatorname{Hom}(\mathbf{X}, \mathbf{Y})$;

3) $\delta(f, h)=\delta\left(f, h^{\prime}\right)$ для любых $h, h^{\prime} \in \operatorname{Hom}(\mathbf{X}, \mathbf{Y})$;

4) $\sum_{0 \neq a \in \mathbf{X}}\left(P\left(f a^{+}-f=h(a)\right)-|\mathbf{Y}|^{-1}\right)=0$ для любого $h \in \operatorname{Hom}(\mathbf{X}, \mathbf{Y})$;

5) $\sum_{0 \neq a \in \mathbf{X}}\left(P\left(f a^{+}-f=h(a)\right)-|\mathbf{Y}|^{-1}\right)=\sum_{0 \neq a \in \mathbf{X}}\left(P\left(f a^{+}-f=h^{\prime}(a)\right)-|\mathbf{Y}|^{-1}\right)$ для любых $h, h^{\prime} \in \operatorname{Hom}(\mathbf{X}, \mathbf{Y})$;

6) $\sum_{0 \neq c \in \mathbf{Z}_{p}}\left(P\left(f(c a)^{+}-f=c y\right)-|\mathbf{Y}|^{-1}\right)=0$ для любых $a \in \mathbf{X} \backslash\{0\}, y \in \mathbf{Y}$;

7) $\sum_{0 \neq c \in \mathbf{Z}_{p}}\left(P\left(f(c a)^{+}-f=c y\right)-|\mathbf{Y}|^{-1}\right)=\sum_{0 \neq c \in \mathbf{Z}_{p}}\left(P\left(f(c a)^{+}-f=c y^{\prime}\right)-|\mathbf{Y}|^{-1}\right)$ для любых $a \in \mathbf{X} \backslash\{0\}, y, y^{\prime} \in \mathbf{Y}$.

Утверждение 1 означает, что свойство абсолютной негомоморфности (т. е. «бентовости») наследуется гомоморфными образами, а также что бентфункции не имеют нетривиальных гомоморфных образов с $\left|\mathbf{X}^{\prime}\right|<|\mathbf{X}|$. Это справедливо и для свойства абсолютной минимальности.

Следствие 8 ([11]). Если в примарном случае $f \in A M\left(\mathbf{Y}^{\mathbf{X}}\right), f^{\prime}: \mathbf{X}^{\prime} \rightarrow \mathbf{Y}^{\prime}-$ гомоморфный образ функиии $f$ u $\left|\mathbf{Y}^{\prime}\right|>1$, то $\left|\mathbf{X}^{\prime}\right|=|\mathbf{X}| u f^{\prime} \in A M\left(\mathbf{Y}^{\prime} X^{\prime}\right)$.

Заметим, что следствие 8 для $\mathbf{Y}^{\prime}=\mathbf{Z}_{p}$ было доказано В. А.Шишкиным в 2008 году (результат не опубликован).

Следующая теорема обобщает хорошо известный для булева случая факт (см., например, [9] или формулу (25)) на двоичный и троичный случаи.

Теорема 4 ([11]). Для р-примарного случая с $p \in\{2,3\}$ имеет место совпадение $A N\left(\mathbf{Y}^{\mathbf{X}}\right)=A M\left(\mathbf{Y}^{\mathbf{X}}\right)$.

В дальнейшем представляется целесообразным развитие изложенных результатов по следующим направлениям.

1) Одни и те же функции из множества $X$ в множество $Y$ можно рассматривать для различных групповых операций на этих множествах и, тем самым, оценивать введенные выше характеристики отличия одних и тех же функиий от различных множеств гомоморфизмов. Например, $\left|\operatorname{Hom}\left(\mathbf{Z}_{p^{n}}, Z_{p}\right)\right|=p$, a $\left|\operatorname{Hom}\left(\left(Z_{p}\right)^{n}, Z_{p}\right)\right|=p^{n}$. Вопрос об оптимальности выбора групповых операций для заданного класса функций представляет значительный интерес. 
2) Изучение предложенных обобщений: максимально негомоморфных функций, минимально близких к гомоморфизмам функций (прежде всего, взаимно однозначных).

3) Обобщение теоремы 4 о совпадении классов абсолютно минимальных функций и бент-функций на любой примарный случай.

В заключение автор выражает глубокую благодарность своему Учителю - Михаилу Михайловичу Глухову, в том числе за его ценные замечания и советы, существенно повлиявшие на содержание этой работы.

\section{Список литературы}

[1] Амбросимов А.С., “Свойства бент-функций $q$-значной логики над конечными полями”, Дискретная математика, $6: 3$ (1994), 50-60.

[2] Golomb S.W., "On the classification of Boolean functions", IRE Trans. Circuit Theory, 1:6 (1959), 10-27.

[3] Кузьмин А.С., Нечаев А.А., Шишкин В.А., “Бент- и гипербент-функции над конечным полем", Труды по дискретной математике, 10 (2007), 97-122.

[4] Kumar P.V., Scholts R.A., Welch L.R., "Generalized bent functions and their properties", $J$. Comb. Theory, Ser. A, 40:1 (1985), 90-107.

[5] Кэртис Ч., Райнер И., Теория представлений конечных групп и ассочиативных алгебр : М.: Издательство «Наука», 1969, 668 с.

[6] Логачев О.А., Сальников А.А., Ященко В.В., “Бент-функции на конечной абелевой группе”, Дискретная математика, 9:4 (1997), 3-20.

[7] Nyberg K., "Perfect nonlinear S-boxes", Lect. Notes Comput. Sci., 547, 1991, 378-386.

[8] Pott A., "Nonlinear functions in abelian groups and relative difference sets", Discrete Applied Mathematics, 138 (2004), 177-193.

[9] Rothaus O.S., “On «bent» functions”, J. Comb. Theory, Ser. A, $20: 3$ (1976), 300-305.

[10] Солодовников В.И., “Бент-функции из конечной абелевой группы в конечную абелеву группу”, Дискретная математика, 14:1 (2002), 99-113.

[11] Солодовников В.И., “О примарных функциях, минимально близких к линейным”, Maтематические вопросы криптографии, $2: 4$ (2011), 97-108.

[12] Солодовников В.И., “О совпадении класса бент-функций с классом функций, минимально близких к линейным”, Прикладная дискретная математика, № 3 (2012), 25-33.

[13] Токарева Н.Н., Нелинейные булевы функции: бент-функиии и их обобщения: Saarbrucken, Germany: LAP LAMBERT Academic Publishing, 2011, 180 c. 